\title{
The Clinicopathologic Heterogeneity of Grade 3 Gastroenteropancreatic Neuroendocrine Neoplasms: Morphological Differentiation and Proliferation Identify Different Prognostic Categories
}

\author{
Massimo Milione $^{a}$ Patrick Maisonneuve ${ }^{c}$ Francesca Spadad $^{d}$ Alessio Pellegrinellia \\ Paola Spaggiari $^{f}$ Luca Albarellog $^{9}$ Eleonora Pisa ${ }^{\mathrm{e}}$ Massimo Barberis ${ }^{\mathrm{e}}$ \\ Alessandro Vanoli ${ }^{\text {h }}$ Roberto Buzzoni ${ }^{\text {b }}$ Sara Pusceddu ${ }^{\text {b }}$ Laura Concas $^{b}$ \\ Fausto Sessa $^{\mathrm{i}}$ Enrico Solcia $^{\mathrm{h}}$ Carlo Capella $^{\mathrm{i}}$ Nicola Fazio $^{d}$ Stefano La Rosa ${ }^{\mathrm{j}}$ \\ ${ }^{a}$ Anatomic Pathology, Department of Pathology and Laboratory Medicine, and ${ }^{\mathrm{b}}$ Departement of Medical Oncology, IRCCS \\ Foundation National Cancer Institute, ' Division of Epidemiology and Biostatistics, ${ }^{\mathrm{d}}$ Gastrointestinal Medical Oncology and \\ Neuroendocrine Tumors Unit, and e Division of Pathology, European Institute of Oncology (IEO), ${ }^{f}$ Cancer Center Humanitas, \\ and ${ }^{g}$ Pathology Unit, San Raffaele Hospital, Milan, ${ }^{\mathrm{h}}$ Fondazione IRCCS Policlinico San Matteo and Department of Molecular \\ Medicine, University of Pavia, Pavia, 'Department of Surgical and Morphological Sciences, University of Insubria, and \\ 'Department of Pathology, Ospedale di Circolo, Varese, Italy
}

\section{Key Words}

Neuroendocrine carcinoma · Morphology · Proliferation ·

Classification · Prognosis

\begin{abstract}
Background/Aims: Gastroenteropancreatic (GEP) neuroendocrine carcinomas (NECs) are defined as neuroendocrine neoplasms (NENs) with a Ki-67 index $>20 \%$ according to the 2010 WHO classification. Some reports suggest that this category is heterogeneous. We retrospectively studied a series of 136 patients affected by grade 3 GEP-NECs with the aim to clarify the prognostic role of tumor morphological differentiation, proliferation, defect in mismatch repair proteins (MMRd), CD117 expression, and site of origin. The primary endpoint was the correlation between these parameters and the overall survival (OS). Methods: Univariate and multivariable Cox proportional hazards regression analyses were
\end{abstract}

used to assess the prognostic significance of various clinical and histopathologic features. Results: With a median followup of 81 months, the median OS was 12.9 months. At multivariate analysis, morphological differentiation, Ki-67 index, MMRd, stage, and CD117 expression were independent prognostic markers in NECs. Three different prognostic categories of NECs were identified according to the degree of morphologic differentiation (well vs. poorly differentiated) and $\mathrm{Ki}-67$ index ( $<55 \%$ vs. $\geq 55 \%$ ). On this basis, median OS was 43.6 months in well-differentiated neoplasms with a Ki67 index 20-55\% (named type A), 24.5 months in poorly differentiated neoplasms with a Ki-67 index 20-55\% (type B), and 5.3 months ( $p<0.0001$ ) in poorly differentiated neoplasms with a Ki-67 index $\geq 55 \%$ (type C). Conclusions: The present study suggests that GEP-NECs represent a heterogeneous group of neoplasms which can be better classified in different prognostic categories using both tumor morphology and Ki-67 index.

(c) 2016 S. Karger AG, Basel

\section{KARGER}

(c) 2016 S. Karger AG, Basel

E-Mail karger@karger.com

www.karger.com/nen
Massimo Milione

Anatomic Pathology 1, Department of Pathology and Laboratory Medicine Fondazione IRCCS Istituto Nazionale dei Tumori, Via G. Veneziana, 1 IT-20133 Milan (Italy)

E-Mail massimo.milione@ istitutotumori.mi.it 


\section{Introduction}

Gastroenteropancreatic neuroendocrine neoplasms (GEP-NENs) are a heterogeneous group of neoplastic proliferations encompassing a wide spectrum of entities ranging from low proliferative well-differentiated neuroendocrine tumors (NETs) to high-grade poorly-differentiated neuroendocrine carcinomas (NECs). According to the 2010 WHO classification of tumors of the digestive system, GEP-NENs are currently classified as follows: (a) grade 1 NETs with less than 2 mitoses per 10 high-power fields (HPF) and a Ki-67 index $\leq 2 \%$; (b) grade 2 NETs with mitotic count between 2 and 20 mitoses per $10 \mathrm{HPF}$ and/or a Ki-67 index between 3 and 20\%; (c) grade 3 NECs showing more than 20 mitoses per $10 \mathrm{HPF}$ and/or a Ki-67 index $>20 \%$ [1].

GEP-NECs are rare cancers and are considered neoplasms associated to an unfavorable prognosis. However, to the best of our knowledge, literature does not report of detailed studies comparing the tumor morphological characteristics and survival for GEP-NECs according to different anatomical sites of origin (esophagus, stomach, pancreas, colon-rectum, biliary tract). Moreover, although GEP-NECs, as classified according to the 2010 WHO classification, have traditionally been considered as a unique clinical entity, recent studies have suggested a subclassification, mainly according to Ki-67 index, of different prognostic categories with distinct response to medical treatments $[2,3]$. Recent data have also suggested tumor morphology as an important prognostic player in determining survival in patients with GEP-NENs showing a Ki-67 index >20\% [3-6]. However, the relationship between tumor morphology and proliferation and their reciprocal role in determining patients' survival is yet to be unveiled [2-7]. The introduction of a new category of GEP-NENs characterized by well-differentiated tumor morphology and Ki-67 index $>20 \%$, named grade 3 welldifferentiated NETs, has been proposed $[3,8]$, but it currently represents a matter of debate $[3-5,9,10]$. Mismatch repair deficiency (MMRd), i.e. lack of at least one mismatch repair protein at the immunohistochemical analysis, with consequent microsatellite instability has also been recently proposed as a prognostic marker identifying a subset of GEP-NECs showing a longer survival than expected, yet needing further validation $[11,12]$.

The aim of this study was to analyze a large series of poorly differentiated and/or grade 3 (Ki-67 >20\%) GEPNECs in order to (a) detect mitotic and proliferative (Ki67) indices to distinguish different prognostic groups of GEP-NECs; (b) evaluate the prognostic value of tumor morphology in terms of well-differentiated and poorly differentiated neoplasms; (c) identify the prognostic relevance of the specific origin of the neoplasms, and (d) define the prognostic relevance of MMRd.

\section{Materials and Methods}

\section{Case Selection and Study Design}

The surgical pathology and clinical database related to the period 1995-2011 were retrospectively analyzed in six Italian institutions: National Cancer Institute, Milan; European Institute of Oncology, Milan; Humanitas Hospital, Rozzano; IRCCS Policlinico San Matteo, Pavia; San Raffaele Hospital, Milan; Ospedale di Circolo, Varese. Patients with one of the following diagnoses were included: small-cell carcinoma, large-cell neuroendocrine carcinoma, poorly differentiated neuroendocrine carcinoma, neuroendocrine carcinoma, and grade 3 neuroendocrine neoplasm of the GEP system.

Three hundred and fifty formalin-fixed paraffin embedded tissue samples from GEP-NENs, including surgical and biopsy specimens, were retrieved and morphologically reviewed by a team of five NEN expert pathologists (M.M., S.L.R., C.C., P.S., L.A.) blinded to clinical and follow-up information. Consensus meetings involving all the participants were held from December 2013 to December 2014. Each meeting discussed a maximum of ten cases: slides were reviewed under a multiheaded microscope, and final pathologic diagnosis was based on unanimous or majority consensus among the NEN expert pathologists using the following criteria: (a) tumor morphology (well and poorly differentiated); (b) immunoreactivity for general neuroendocrine markers (synaptophysin, chromogranin A; for cases in which chromogranin A or synaptophysin was negative, CD56 or PGP 9.5 were added as second-line neuroendocrine markers; (c) mitotic index $>20$ mitoses $\times 50 \mathrm{HPF}$ and/or Ki-67 index $>20 \%$. Patients with a history of NECs located in the lung or in other non-GEP sites and patients with mixed adenoneuroendocrine carcinomas were excluded. Overall, 136 patients met all the criteria and were selected for inclusion. This study was performed according to the clinical standards of the 1975 and 1983 Declaration of Helsinki and was approved by the Ethical Committee of the Ospedale di Circolo of Varese (No. 0008465).

\section{Histological Analysis}

The 136 selected NEC samples were also analyzed for morphological parameters such as histologic subtype (small versus large cell), necrosis, lymphovascular invasion (evaluated in both HEand CD31-stained sections), perineural invasion, absence of proteins involved in DNA mismatch repair, tumoral lymphocytic infiltration, tumor size, level of gut wall invasion for gastrointestinal neoplasms or peripancreatic infiltration for pancreatic neoplasms, and staging according to AJCC/UICC [13] and ENETS [14].

Morphologically, well-differentiated and poorly differentiated NENs were also distinguished separately according to different criteria. In details, well-differentiated NETs were characterized by microlobular, microacinar or thinly trabecular structures formed by relatively monomorphic, regularly arranged cells with fine chromatin and inconspicuous nucleoli. In some cases, tumors showed larger and more disordered cell aggregates, with larger,
Milione et al. 

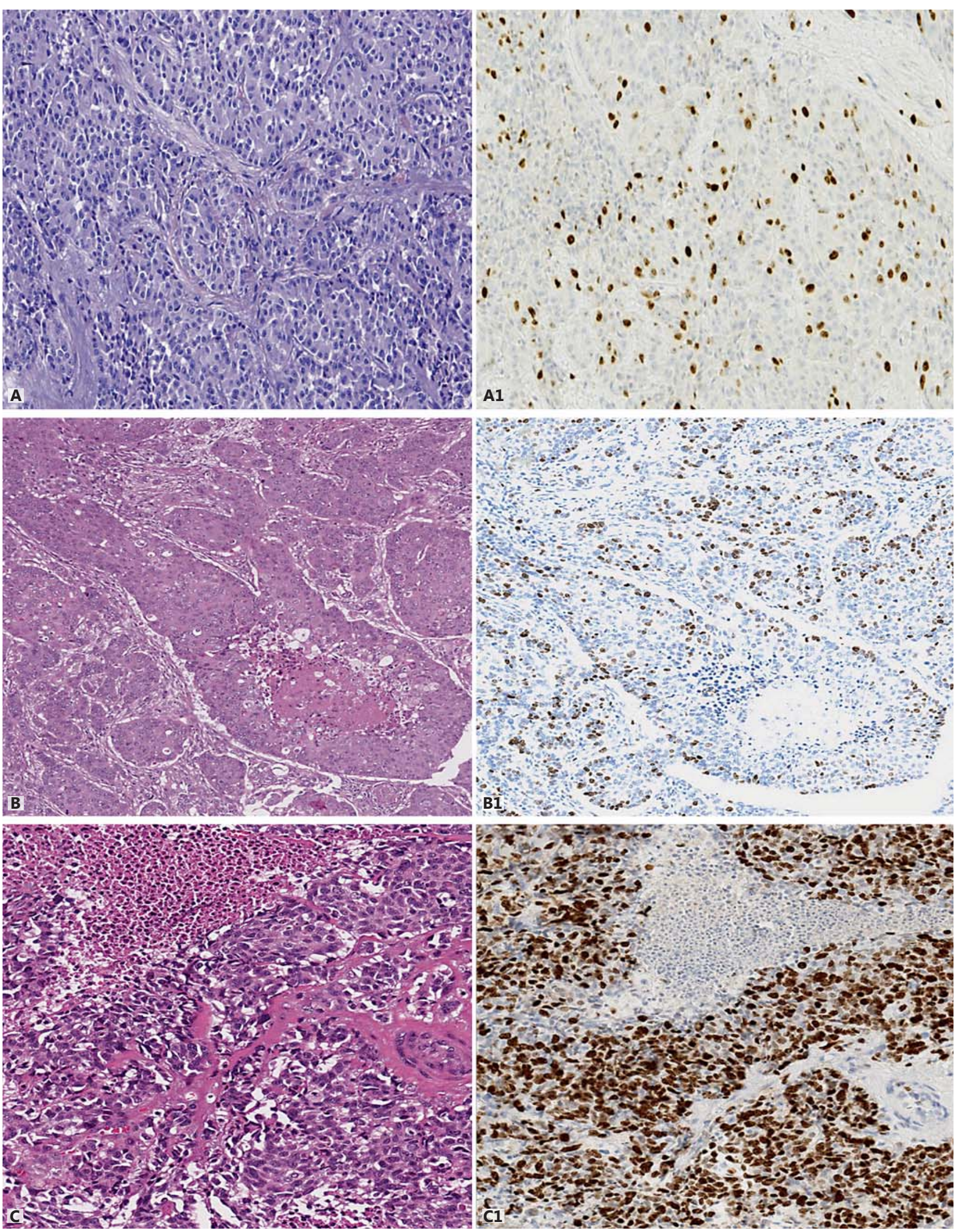

(For legend see next page.) 
more crowded and poorly aligned nuclei, with moderate atypia, less regularly distributed chromatin, more evident nucleoli, sometimes with focal necrosis. These latter morphological features were proposed for the definition of morphologically moderately differentiated NETs [15]. Poorly differentiated NECs showed poorly formed, large trabeculae and solid nests or diffuse sheets of cells frequently showing multiple necrotic foci or large and confluent areas of 'geographic chart' necrosis and brisk mitotic activity. Moreover, based on the morphological features of the neoplastic cells, they were further subdivided into small- or large-cell subtypes. In details, small-cell carcinomas were composed of small to medium-sized (2-4 times of lymphocyte size), round to oval cells with scant cytoplasm and hyperchromatic nuclei with indistinct nucleoli. On the other hand, large-cell subtypes were composed of large cells with vesicular nuclei showing prominent nucleoli and abundant eosinophilic cytoplasm.

The Ki-67 index was defined using the MIB antibody as a percentage of 500-2,000 cells counted in areas of strongest nuclear labelling ('hot spots') [1]; mitotic count was evaluated in at least 50 HPF $\left(1 \mathrm{HPF}=2 \mathrm{~mm}^{2}\right)[1]$. Based on tumor morphological differentiation and Ki-67 index, the following three categories were defined: (a) type A NECs showing a Ki-67 index of $20-55 \%$ and welldifferentiated morphology; (b) type B NECs with a Ki-67 index of $20-55 \%$ and poorly differentiated morphology; (c) type C NECs with poorly differentiated morphology and a Ki-67 index $\geq 55 \%$ (fig. 1).

In addition to common neuroendocrine markers and Ki-67 immunohistochemistry, the following immunostainings were also performed: CD117 (also known as cKit), recently proposed as marker of worse prognosis [11] (dilution 1:400; code No. A450229), hMLH1 (1:50; M3640), hMSH2 (1:100; M3639), hMSH6 (1:200; M3646), hPMS2 (1:100; M3647). All antibodies were purchased from Dako, Glostrup, Denmark. MMRd was established according to the criteria reported by Chiaravalli et al. [16].

\section{Statistical Analysis}

Clinical and pathologic characteristics of patients were stratified according to tumor type: type A NECs versus type B NECs versus type $C$ NECs. Differences in frequencies were assessed with the $\chi^{2}$ or the Fisher exact test. Overall survival (OS) was the primary study endpoint and was assessed from the time of the diagnosis to the time of death or last follow-up. Survival curves were drawn according to the Kaplan-Meier method, and differences be-

Fig. 1. A, A1 Type A neuroendocrine carcinoma. The neoplasm is composed of relatively monomorph neoplastic cells, arranged in regular nests without necrosis, with nuclei showing finely dispersed chromatin without evidence of severe atypia. The proliferation index assessed with MIB1-Ki-67 is less than 55\% (A1). B, B1 Type B neuroendocrine carcinoma. The neoplasm is composed of atypical neoplastic cells, distributed in irregular structures for size and shape, with prominent nucleoli and intratumoral necrotic foci. The proliferation index assessed with MIB1-Ki-67 is less than $55 \%$ (B1). C, C1 Type C neuroendocrine carcinoma. The neoplasm is composed of large and intermediate neoplastic cells, with atypical nuclei with prominent nucleoli. Abundant intratumoral necrosis is evident. The proliferation index assessed with MIB1$\mathrm{Ki}-67$ is $55 \%$ or more (C1). tween groups (CD117, differentiation, mitotic count, Ki-67 index, microsatellite instability, lymphocytic infiltrate and angioinvasion) were assessed with the log-rank test. The number of patients at risk and the proportion of patients surviving at different time points are presented with respective $95 \%$ confidence interval (CI). Univariate and multivariable Cox proportional hazards regression analysis was used to assess the prognostic significance of the various clinical and histopathologic characteristics on OS. Data analysis was performed using the SAS software (version 9.2, Cary, N.C., USA). All tests were two-sided, and p values $<0.05$ were considered statistically significant.

\section{Results}

\section{Clinicopathologic Features}

Table 1 summarizes the main clinicopathologic features of the 136 patients enrolled in the study. Of those, $81(59.5 \%)$ patients were males, and the mean age at diagnosis was 64 years. Ninety-four cases (69.1\%) were represented by surgically resected specimens, and 42 cases (30.9\%) by biopsy specimens. One hundred and four samples $(76.5 \%)$ were related to the primary carcinoma, while $32(23.5 \%)$ to liver metastases from unresectable primary GEP-NECs. Primary tumor locations and histological details are summarized in table 1 . Based on tumor morphological differentiation and Ki-67 index (see Materials and Methods), we identified 24 type A NECs, 30 type B NECs and 82 type C NECs. A detailed site distribution of type $\mathrm{A}$, type $\mathrm{B}$, and type $\mathrm{C}$ NECs is provided in online supplementary table S1 (for all online suppl. material, see www.karger.com/doi/10.1159/000445165).

Males were affected more frequently than females with a male/female ratio of 1.47 . The most frequent primary sites for NECs were more frequent in the colon-rectum, pancreas and stomach. Interestingly, among the 24 type A NECs, 11 were located in the pancreas, whereas the remaining 13 cases were more or less equally distributed among the colon, ileum-cecum/appendix and stomach. No type A NECs were observed in the esophagus and duodenum. Type B NECs were found in the pancreas, stomach and colon, while they were rarer in other sites. Type C NECs were more frequently observed in the colon-rectum and stomach than in other GEP sites. CD117 immunoreactivity was more frequently observed in type $\mathrm{B}$ and C NECs (25.0 and $25.4 \%$ of cases, respectively) than in type A (12.5\%), while MMRd was more frequently observed in type A (28.6\% of cases) than in type B and C NECs (15.4 and $4.3 \%$ of cases, respectively). Angioinvasion was a frequent finding in type C NECs (77.4\%), while it was less frequent in type B (48.0\%) and type A (36.8\%). 


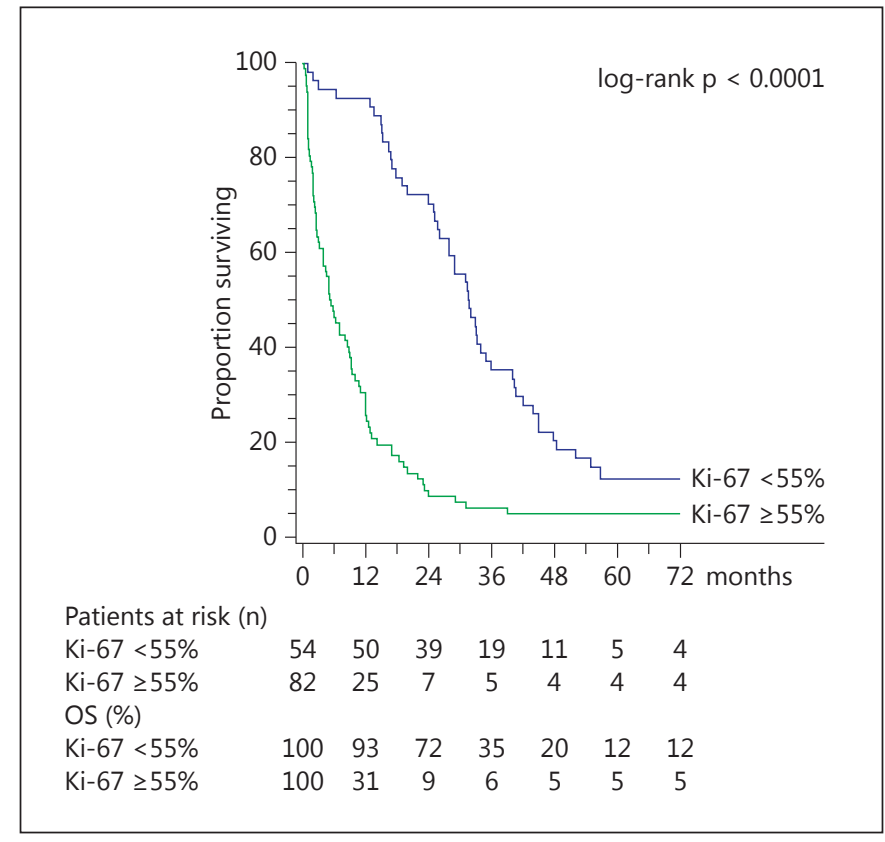

Fig. 2. OS of 136 patients with NEC according to Ki-67 proliferative index.

$60.3 \%$ of cases were at stage IV at the time of diagnosis, including $15 / 24$ (62.5\%) type A, 14/30 (46.7\%) type B, and 53/82 (64.6\%) type C NECs.

\section{Survival Analysis}

The median follow-up was 81 months (range $<1-230$ months). The median OS was 12.9 months (95\% CI 9.317.1). The prognostic meaning of several morphological and proliferative parameters is reported in table 2. Utilizing the statistical criteria defined by Sylvain and Richard [17], we performed a study on mitotic count and we showed that the cut-off of 30 mitoses (online suppl. fig. $\mathrm{S} 1 \mathrm{~A}$ ) was the best predictor of OS. In our cases, the distribution of Ki-67 index values (online suppl. fig. S1B) allowed confirming the cut-off of $55 \%$ proposed by Sorbye et al. [2]. Moreover, both Ki-67 and mitotic count (30 mitoses) correlated with OS. At univariate analysis, poorly differentiated tumor morphology, mitotic count $>30$ mitoses/10 HPF, Ki-67 index $\geq 55 \%$, absence of MMRd, CD117 expression, angioinvasion, mid-hindgut origin, and stage IV correlated with worse prognosis. Moreover, poorly differentiated morphology, Ki-67 index $\geq 55 \%$, absence of MMRd, mid-hindgut origin, and stage IV were confirmed as negative independent prognostic markers at multivariate analysis (table 2). In addition to the present multivariable analysis, we also performed an alterna-
Table 1. Clinicopathologic features of 136 patients with NEC

\begin{tabular}{|c|c|c|c|c|}
\hline & All & Type A & Type B & Type C \\
\hline Total & 136 & 24 & 30 & 82 \\
\hline \multicolumn{5}{|l|}{ Gender } \\
\hline Men & 81 & 15 & 15 & 51 \\
\hline Women & 55 & 9 & 15 & 31 \\
\hline \multicolumn{5}{|l|}{ Tumor site } \\
\hline Esophagus & 5 & 0 & 1 & 4 \\
\hline Stomach & 28 & 5 & 6 & 17 \\
\hline Duodenum & 5 & 0 & 3 & 2 \\
\hline Ileum-cecum/appendix & 17 & 4 & 3 & 10 \\
\hline Colon-rectum & 46 & 4 & 8 & 34 \\
\hline Pancreas & 33 & 11 & 9 & 13 \\
\hline Gallbladder & 2 & 0 & 0 & 2 \\
\hline \multicolumn{5}{|l|}{ Mitotic count/10 HPF } \\
\hline$<20$ & 44 & 17 & 24 & 3 \\
\hline $20-29$ & 18 & 7 & 3 & 8 \\
\hline$\geq 30$ & 74 & 0 & 3 & 71 \\
\hline \multicolumn{5}{|l|}{ CD117 } \\
\hline Negative & 63 & 7 & 12 & 44 \\
\hline Positive & 20 & 1 & 4 & 15 \\
\hline Missing & 53 & 16 & 14 & 23 \\
\hline \multicolumn{5}{|l|}{ MMRd } \\
\hline Absent & 61 & 5 & 11 & 45 \\
\hline Present & 6 & 2 & 2 & 2 \\
\hline Missing & 69 & 17 & 17 & 35 \\
\hline \multicolumn{5}{|l|}{ Angioinvasion } \\
\hline Absent & 39 & 12 & 13 & 14 \\
\hline Present & 67 & 7 & 12 & 48 \\
\hline Missing & 30 & 5 & 5 & 20 \\
\hline \multicolumn{5}{|l|}{ Lymphocytic infiltration } \\
\hline Absent & 94 & 14 & 19 & 61 \\
\hline Present & 29 & 7 & 3 & 19 \\
\hline Missing & 13 & 3 & 8 & 2 \\
\hline \multicolumn{5}{|l|}{ Stage (ENETS) } \\
\hline I-II & 9 & 2 & 1 & 6 \\
\hline III & 45 & 7 & 15 & 23 \\
\hline IV & 82 & 15 & 14 & 53 \\
\hline
\end{tabular}

tive and explorative multivariable analysis without Ki-67, in which mitotic count resulted as an independent prognostic factor $(\mathrm{p}=0.01)$ with the cut-off of 30 mitoses/50 HPF.

The 55\% cut-off of the Ki-67 index was statistically correlated with OS ( $p<0.0001$; fig. 2). However, the prognostic stratification of patients was greatly improved when they were grouped considering both the Ki-67 index ( $<55$ vs. $\geq 55 \%$ ) and the degree of morphologic differentiation (well differentiated vs. poorly differentiated; fig. 3). The median OS was 43.6 months (95\% CI 33.054.9) in type A NECs, 24.5 months (95\% CI 16.9-29.0) in 
Table 2. Predictors of survival at univariate and multivariable analysis

\begin{tabular}{|c|c|c|c|}
\hline & & HR (95\% CI) & $\mathrm{p}$ value \\
\hline \multicolumn{4}{|l|}{ Univariate analysis } \\
\hline Site & Mid-hindgut vs. foregut & $1.55(1.10-2.21)$ & 0.01 \\
\hline CD117 & Positive vs. negative & $1.42(0.85-2.39)$ & 0.18 \\
\hline Differentiation & $\mathrm{PD}$ vs. WD/MD & $4.06(2.43-6.78)$ & $<0.0001$ \\
\hline Mitotic count & $\geq 30$ vs. $<30$ & $3.01(2.09-4.34)$ & $<0.0001$ \\
\hline $\mathrm{Ki}-67$ & $\geq 55 \%$ vs. $<55 \%$ & $3.35(2.30-4.88)$ & $<0.0001$ \\
\hline MMRd & Present vs. absent & $0.26(0.11-0.67)$ & 0.005 \\
\hline Lymphocytic infiltration & Present vs. absent & $1.06(0.69-1.62)$ & 0.79 \\
\hline Angioinvasion & Present vs. absent & $1.64(1.09-2.47)$ & 0.02 \\
\hline Stage (ENETS) & Stage IV vs. I-III & $1.45(1.02-2.07)$ & 0.04 \\
\hline \multicolumn{4}{|l|}{ Multivariable analysis } \\
\hline Site & Mid-hindgut vs. foregut & $1.78(1.17-2.70)$ & 0.008 \\
\hline CD117 & Positive vs. negative & $2.25(1.26-4.00)$ & 0.006 \\
\hline Differentiation & $\mathrm{PD}$ vs. WD/MD & $2.83(1.47-5.47)$ & 0.002 \\
\hline Mitotic count & $\geq 30$ vs. $<30$ & $1.06(0.56-2.01)$ & 0.87 \\
\hline $\mathrm{Ki}-67$ & $\geq 55 \%$ vs. $<55 \%$ & $2.10(1.00-4.44)$ & 0.05 \\
\hline MMRd & Present vs. absent & $0.16(0.06-0.45)$ & 0.0005 \\
\hline Lymphocytic infiltration & Present vs. absent & $1.05(0.65-1.69)$ & 0.85 \\
\hline Angioinvasion & Present vs. absent & $1.57(0.98-2.52)$ & 0.06 \\
\hline Stage (ENETS) & Stage IV vs. I-III & $1.59(1.07-2.38)$ & 0.02 \\
\hline
\end{tabular}

Hazards ratio (HR) and 95\% CI obtained from univariate and multivariable COX proportional hazards regression models. $\mathrm{PD}=$ Poorly differentiated; $\mathrm{MD}=$ moderately differentiated; $\mathrm{WD}=$ well differentiated.

Table 3. Comparison of overall survival of patients with pancreatic NET grade 2 (G2), gastric NET G2, type A, type B, and type C NECs

\begin{tabular}{|c|c|c|c|c|}
\hline & 12-month & 24-month & 36-month & 72-month \\
\hline Pancreatic NET G2 ${ }^{\mathrm{a}}$ & 90.7 & 86.7 & 82.5 & 72.8 \\
\hline Gastric NET G2b & 92.3 & 71.2 & 71.2 & 71.2 \\
\hline Type A NEC & 100 & 96.8 & 67.7 & 36.9 \\
\hline Type B NEC & 83.7 & 42.9 & 14.3 & 4.1 \\
\hline Type C NEC & 35.9 & 11.5 & 6.1 & 3.8 \\
\hline
\end{tabular}

${ }^{\mathrm{a}}$ Data from [18]; ${ }^{\mathrm{b}}$ data from [16].

type B NECs, and 5.3 months (95\% CI 3.3-8.9) in type C NECs $(\mathrm{p}<0.0001)$. Survival rates at different time points are provided in table 3 . The hazard ratio for death was 0.46 (95\% CI $0.30-0.71 ; \mathrm{p}=0.0004)$ for patients with type $\mathrm{B}$ and 0.18 (95\% CI 0.11-0.31; $<<0.0001)$ for those with type A as compared to type C.

Interestingly, the site of origin was statistically correlated with prognosis at univariate analysis and confirmed as an independent predictor at multivariate analysis (table 2). In particular, midgut and/or hindgut sites of origin correlated with a worse survival as compared with foregut [16] (fig. 4). A detailed comparison of survivals in relation to the site of origin is provided in online supplementary figure S2.

\section{Medical Treatment}

Information on medical therapy was available for 110 out of $136(80.9 \%)$ patients, 59 of whom received a firstline therapy with cisplatin/carboplatin+etoposide, 31 with other platinum-based chemotherapies, 12 with non-platinum-based chemotherapy and 8 received other non-cytotoxic therapy. The significant difference in terms of OS between type A, type B and type C was confirmed in these 59 patients (online suppl. fig. S3). When the group of patients receiving cisplatin/ carboplatin+etoposide was compared with the group of patients who had received other chemotherapies, no statistically significant difference in OS $(\mathrm{p}=0.32)$ was observed (online suppl. fig. S4).
90 


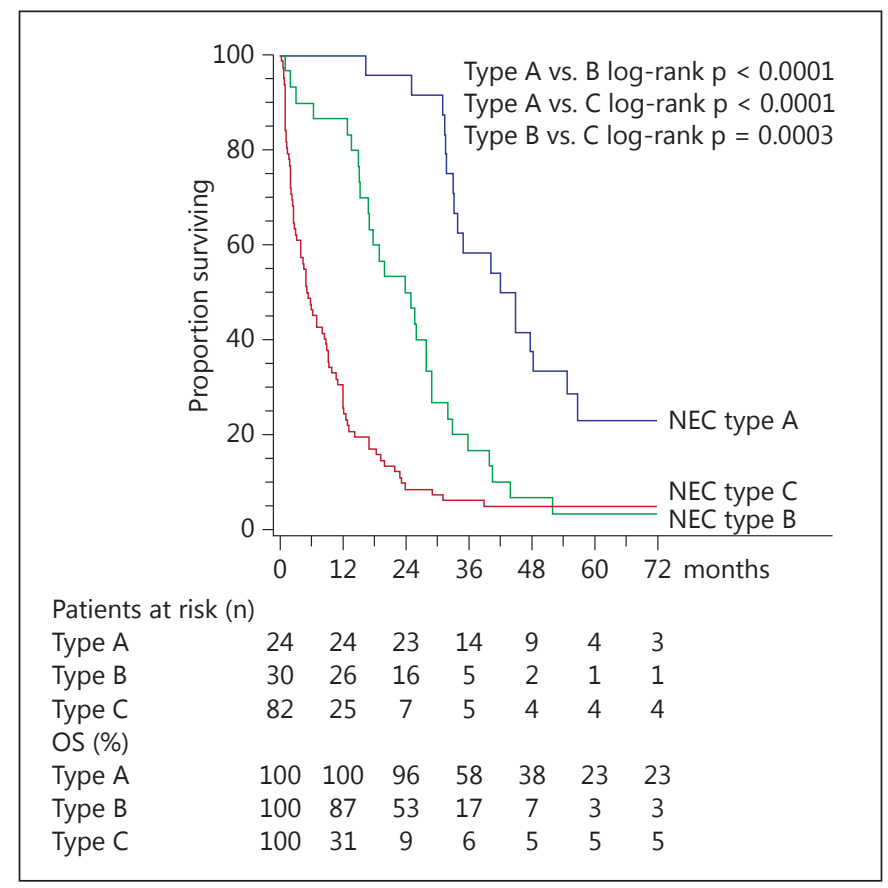

Fig. 3. OS of 136 patients with NEC according to subtype.

\section{Discussion}

Our study demonstrates that GEP-NECs represent a heterogeneous group of neoplasms which can be better classified in different prognostic categories using both tumor morphology and Ki-67 index. In details, well-differentiated tumor morphology is an independent prognostic factor for GEP-NENs with a Ki-67 index of $20-55 \%$, while the 55\% Ki-67 cut-off is an independent prognostic factor for GEP-NENs with poorly differentiated tumor morphology. By combining tumor morphological differentiation and Ki-67 index, we were able to better define three prognostic categories: type $\mathrm{A}$, type $\mathrm{B}$, and type $\mathrm{C}$ GEP-NECs. The prognostic value of these three categories was the same, regardless of the medical treatment and the type of chemotherapy received. These findings better describe the proliferative categories proposed by Sorbye et al. [2], where tumor morphology was not considered.

Interestingly, well-differentiated tumor morphology presented a Ki-67 index less than $55 \%$, while cases composed of poorly differentiated cells (type B and type C NECs) showed a Ki-67 index either less or more than $55 \%$. Our observations regarding poorly differentiated NECs revealed that they could be subdivided into two distinct categories based on a Ki-67 cut-off of 55\% (type B

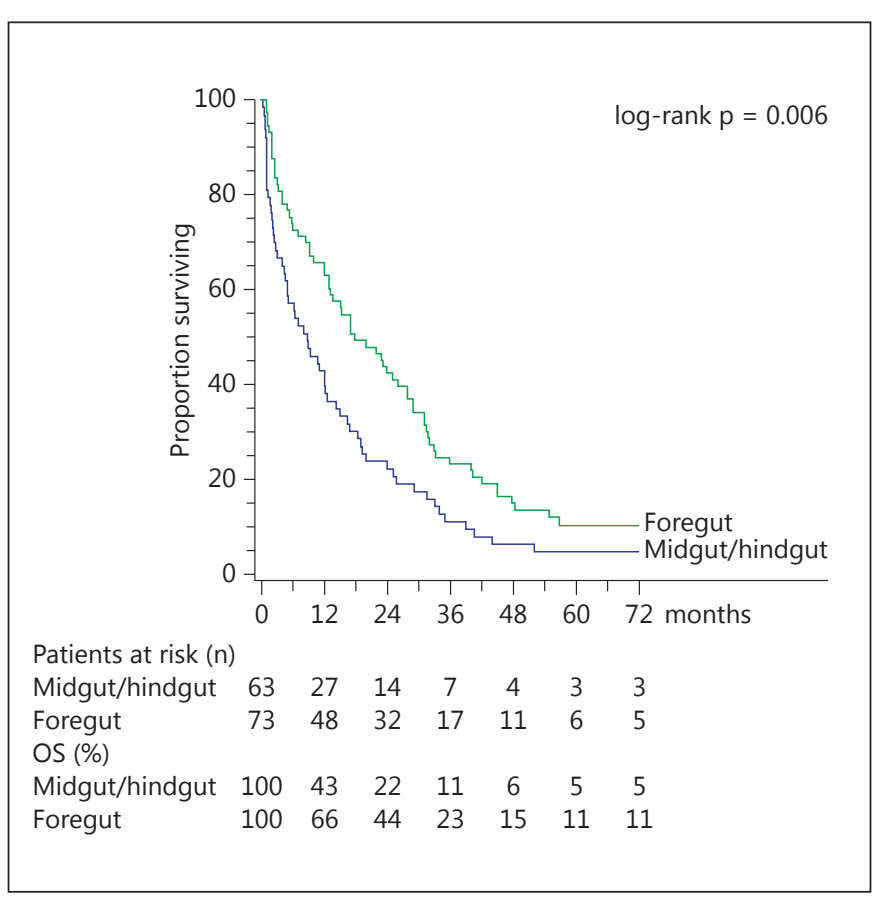

Fig. 4. OS of 136 patients with NEC according to tumor site.

and type C NECs) and deserve a prospective investigation to be further validated.

Recently, Heetfeld et al. [3] proposed 'grade 3 NET' category defining NEN with well-differentiated features and Ki-67 $<55 \%$, which correlates with a significantly different OS compared with poorly differentiated NECs. Similarly, our defined category type A NECs reveals an increased survival than type B and C NECs. In addition, type A NECs show a more aggressive behavior than pancreatic and gastric grade 2 NETs. In detail, the OS of type A NECs is quite similar to the first 2 years of patients with grade 2 NETs $[15,18]$, whereas it becomes strongly worse after the second year of observation (table 3 ). Therefore, type A NECs (or 'grade 3 NETs') should be separated from grade 2 NETs, possibly representing an intermediate category between grade 2 NETs and NECs according to the WHO 2010 classification.

Mitotic count is essential in the description of NEC. We observed that the prognostic role of mitotic count is statistically less important than Ki-67 index. Therefore, we suggest that the mitotic count should be used in absence of adequate material (i.e. bioptic material) to evaluate Ki-67 index. Moreover, our analysis confirms the prognostic role of CD117 [11] as well as the prognostic role of MMRd [11, 12]. Immunohistochemistry for mis- 
match repair proteins to identify this rare but more favorable cancer subtype seems to be important and of prognostic importance. Importantly, our study unveiled the correlation between primary tumor site and prognosis in GEP-NECs, as the outcome of the foregut sites (esophagus, stomach, duodenum, pancreas, and biliary tract) was significantly better.

Our analysis is affected by some weaknesses, including the retrospective design, the type of multicentric series that, although involving some NEN referral centers, could not be representative of the whole Italian NEN population. A further limitation can be the lack of therapeutic correlations; however, an analysis on this topic is ongoing and will be object of future reports. Moreover, the analyzed series is relatively small and suffers from the mix of different sites of origin of the investigated neoplasms.

In conclusion, our retrospective analysis demonstrated that well-differentiated tumor morphology is an independent prognostic factor for GEP-NENs with Ki-67 comprised between $20 \%$ and $-55 \%$ (type A NEC or 'grade 3 NET'), while the 55\% Ki-67 cut-off is an independent prognostic factor for poorly differentiated GEP-NENs (type B and type C NECs).

\section{Acknowledgments}

The authors thank the following persons for their support: Maria Morelli (secretary of the Department of Pathology and Laboratory Diagnostics for literature searches); Giovanna Tagliabue (Lombardy Cancer Registry, Varese Province Cancer Registry Unit Head, Department of Preventive and Predictive Medicine, Fondazione IRCCS Istituto Nazionale dei Tumori, Milan, Italy); Patrizia Gasparini (native English researcher, Tumor Genomics Department of Experimental Oncology) for English revision; Vittorio Perfetti (IRCCS San Matteo, Pavia, Italy); Barbara Martinelli (Ospedale di Circolo, Varese, Italy); Marco Federico Manzoni (San Raffaele Hospital Milan, Milan, Italy); Carlo Carnaghi (Cancer Center Humanitas, Milan, Italy); Claudio Doglioni (San Raffaele Hospital Milan, Milan, Italy).

\section{Disclosure Statement}

The authors declare no conflicts of interest directly relevant to this study.

\section{References}

1 Rindi G, Arnold R, Bosman FT, Capella C, Klimstra DS, Klöppel G, Komminoth P, Solcia E: Nomenclature and classification of digestive neuroendocrine tumours; in Bosman TF, Carneiro F, Hruban RH, Theise ND (eds): World Health Organization Classification of Tumours, Pathology and Genetics of Tumours of the Digestive System. Lyon, International Agency for Research on Cancer (IARC) Press, 2010, pp 10-12.

-2 Sorbye H, Welin S, Langer SW, Vestermark LW, Holt N, Osterlund P, Dueland S, Hofsli E, Guren MG, Ohrling K, Birkemeyer E, ThiisEvensen E, Biagini M, Gronbaek H, Soveri LM, Olsen IH, Federspiel B, Assmus J, Janson ET, Knigge U: Predictive and prognostic factors for treatment and survival in 305 patients with advanced gastrointestinal neuroendocrine carcinoma (WHO G3): the NORDIC NEC study. Ann Oncol 2013;24:152-160.

-3 Heetfeld M, Chougnet CN, Olsen IH, Rinke A, Borbath I, Crespo G, Barriuso J, Pavel M, O'Toole D, Walter T; other Knowledge Network members: Characteristics and treatment of patients with G3 gastroenteropancreatic neuroendocrine neoplasms. Endocr Relat Cancer 2015;22:657-664.

4 Vélayoudom-Céphise FL, Duvillard P, Foucan L, Hadoux J, Chougnet CN, Leboulleux S,
Malka D, Guigay J, Goere D, Debaere T, Caramella C, Schlumberger M, Planchard D, Elias D, Ducreux M, Scoazec JY, Baudin E: Are G3 ENETS neuroendocrine neoplasms heterogeneous? Endocr Relat Cancer 2013;20: 649-657.

5 Basturk O, Yang Z, Tang LH, Hruban RH, Adsay V, McCall CM, Krasinskas AM, Jang KT, Frankel WL, Balci S, Sigel C, Klimstra DS: The high-grade (WHO G3) pancreatic neuroendocrine tumor category is morphologically and biologically heterogenous and includes both well-differentiated and poorly differentiated neoplasms. Am J Surg Pathol 2015;39: 683-690.

- 6 Hijioka S, Hosoda W, Mizuno N, Hara K, Imaoka $\mathrm{H}$, Bhatia V, Mekky MA, Tajika M, Tanaka T, Ishihara M, Yogi T, Tsutumi H, Fujiyoshi T, Sato T, Hieda N, Yoshida T, Okuno N, Shimizu Y, Yatabe Y, Niwa Y, Yamao K: Does the WHO 2010 classification of pancreatic neuroendocrine neoplasms accurately characterize pancreatic neuroendocrine carcinomas? J Gastroenterol 2015;50: 564-565.

7 Welin S, Sorbye H, Sebjornsen S, Knappskog S, Busch C, Oberg K: Clinical effect of temozolomide-based chemotherapy in poorly differentiated endocrine carcinoma after pro- gression on first-line chemotherapy. Cancer 2011;117:4617-4622.

8 Agaimy A, Erlenbach-Wünsch K, Konukiewitz B, Schmitt AM, Rieker RJ, Vieth M, Kiesewetter F, Hartmann A, Zamboni G, Perren A, Klöppel G: ISL1 expression is not restricted to pancreatic well-differentiated neuroendocrine neoplasms, but is also commonly found in well and poorly differentiated neuroendocrine neoplasms of extrapancreatic origin. Mod Pathol 2013;26:9951003.

99 La Rosa S, Sessa F: High-grade poorly differentiated neuroendocrine carcinomas of the gastroenteropancreatic system: from morphology to proliferation and back. Endocr Pathol 2014;25:193-198.

10 Reid MD, Balci S, Saka B, Adsay NV: Neuroendocrine tumors of the pancreas: current concepts and controversies. Endocr Pathol 2014;25:65-79.

11 La Rosa S, Marando A, Furlan D, Sahnane N, Capella C: Colorectal poorly differentiated neuroendocrine carcinomas and mixed adenoneuroendocrine carcinomas: insights into the diagnostic immunophenotype, assessment of methylation profile, and search for prognostic markers. Am J Surg Pathol 2012; 36:601-611. 
12 Sahnane N, Furlan D, Monti M, Romualdi C, Vanoli A, Vicari E, Solcia E, Capella C, Sessa F, La Rosa S: Microsatellite unstable gastrointestinal neuroendocrine carcinomas: a new clinicopathologic entity. Endocr Relat Cancer 2015;22:35-45.

13 Sobin LH, Gospodarowicz MK, Wittekind C: UICC: TNM Classification of Malignant Tumours, 7th ed. Oxford, Wiley-Blackwell, 2009.

14 Rindi G, Klöppel G, Alhman $\mathrm{H}$ aplin M, Couvelard A, de Herder WW, Erikssson B, Falchetti A, Falconi M, Komminoth P, Körner M, Lopes JM, McNicol AM, Nilsson O,
Perren A, Scarpa A, Scoazec JY, Wiedenmann $\mathrm{B}$, et al: TNM staging of foregut (neuro)endocrine tumors: a consensus proposal including a grading system. Virchows Arch 2006;449: 395-401.

15 La Rosa S, Inzani F, Vanoli A, Klersy C, Dainese L, Rindi G, Capella C, Bordi C, Solcia E: Histologic characterization and improved prognostic evaluation of 209 gastric neuroendocrine neoplasms. Hum Pathol 2011;42: 1373-1384.

6 Chiaravalli AM, Furlan D, Facco C, Tibiletti MG, Dionigi A, Casati B, Albarello L, Riva C, Capella C: Immunohistochemical pattern of MSH2/hMLH1 in familial and sporadic colorectal, gastric, endometrial and ovarian carcinomas with instability in microsatellite sequences. Virchows Arch 2001;438:39-48.

17 Sylvain D, Richard S: Flexible regression models with cubic splines. Stats Med 1989;8: 551-561.

18 La Rosa S, Klersy C, Uccella S, Dainese L, Albarello L, Sonzogni A, Doglioni C, Capella C, Solcia E: Improved histologic and clinicopathologic criteria for prognostic evaluation of pancreatic endocrine tumors. Hum Pathol 2009;40:30-40. 